\title{
Zur Zukunft der Demokratie
}

Henk Botha/Nils Schaks/Dominik Steiger

\section{A. Einleitung}

Die Legitimation von Staatsgewalt steckt in der Krise, so liest man es allerorten. Die Zukunft der repräsentativen Demokratie sei unsicher, die Postdemokratie wird ausgerufen. Eine der drängendsten staatsrechtlichen und -politischen Diskussionen der letzten Jahre beschäftigt sich dementsprechend mit der Frage, ob und inwiefern das repräsentative System an seine Grenzen stößt und wie die Demokratie neu zu beleben ist.

Die bisherige Form der parlamentarischen Repräsentation gerät dabei von zwei Seiten unter Druck: Zum einen wird vielfach ein Vertrauensverlust der Repräsentierten in die politische Klasse beklagt. Dieses fehlende Vertrauen ist nicht neu. Zum anderen - und dies ist zumindest in dieser Form neu - besteht ein Ohnmachtsgefühl der Repräsentanten, die sich multiplen und immer komplexeren Anforderungen ausgesetzt sehen, die an sie von der Regierung, den Verfassungsgerichten, den „Märkten“ sowie aufgrund der überstaatlichen Integration herangetragen werden. Das deutet darauf hin, dass Reparaturarbeiten am parlamentarischen System von Nöten sind.

Die Debatte entzündet sich aber nicht nur auf der parlamentarischen Ebene, sondern auch auf der Verwaltungsebene. Angesichts vehementer Proteste gegen Großvorhaben - beispielsweise gegen Stuttgart 21 und gegen Vorhaben im Rahmen der Energiewende - ist die Idee der Partizipation in den Mittelpunkt der Debatte gelangt. Die Politik in Deutschland reagiert - im Bund und in den Ländern in unterschiedlichem Maße - auf die neu entstandene Erwartungshaltung der Bürger durch die Einräumung vereinzelter Formen von Bürgerbeteiligung. Dazu gehören das Standortauswahlgesetz oder die Einführung einer frühen Bürgerbeteiligung im Verwaltungsverfahrensrecht. Es scheint, als ob der Staat seine Regelungsziele immer weniger mittels der altbewährten, aber nicht mehr voll akzeptierten Handlungsformen erreichen kann.

Diese Diskussion beschränkt sich nicht auf Deutschland. Weltweit sehen sich traditionelle Demokratiekonzepte herausgefordert. Bürgerinnen 
und Bürger fühlen sich nicht ausreichend repräsentiert und begehren auf. Auch in Südafrika ist ein solches Phänomen zu beobachten. Hier herrscht große Unzufriedenheit mit dem politischen System und seinen Repräsentanten. Dies äußert sich in zum Teil gewaltsamen Protesten - Südafrika ist hier weltweit führend: Nahezu täglich finden gewaltsame Aktionen statt. Die Ursachen hierfür sind ebenso komplex wie vielschichtig und umfassen eine mangelhafte Daseinsvorsorge (wie z.B. Unterbrechungen in der Stromversorgung), Korruption, Gewalt seitens der Polizei sowie einen Mangel an demokratischer Rechenschaft der lokalen Hoheitsträger. Trotz zahlreicher Unterschiede zwischen Südafrika und Deutschland ist Südafrika als Vergleichsland besonders geeignet, denn es teilt mit Deutschland ein ähnliches politisches System. Die südafrikanische Verfassung, die 2014 ihren 20. Jahrestag feierte, baut - wie das Grundgesetz - auf der Menschenwürde und den Menschenrechten auf und sieht ein föderales parlamentarisches System sowie eine starke Stellung des Verfassungsgerichts vor. Beide Verfassungen beruhen auf Unrechtserfahrungen der Vergangenheit und wollen eine menschliche, rechtsstaatliche und demokratische Gegenwart und Zukunft sichern. Wie derzeit in Deutschland kann sich auch die Regierung in Südafrika auf eine überdeutliche Mehrheit im Parlament stützen. Allerdings wird Deutschland von einer Koalitionsregierung regiert, während in Südafrika eine einzige Partei, der Afrikanische Nationalkongress (ANC), regelmäßig mehr als 60\% der Stimmen auf sich vereinigt; zuletzt anlässlich der Parlamentswahlen im Mai 2014. Die Rechtswissenschaft und die Verfassungsgerichtsbarkeit in beiden Ländern beschäftigen sich mit diesen Entwicklungen und erkennen neue - und jeweils unterschiedliche - Formen demokratischer Partizipation an.

Ziel des hier vorgelegten Tagungsbandes ist es, diesen Wandel der Staatsgewalt und die damit verbundenen Herausforderungen des Demokratieprinzips zu diskutieren und Lösungsansätze zu entwickeln. Nach einer ersten Diagnose des Legitimationsverlustes des repräsentativen Staates (B.) werden Heilungsmöglichkeiten innerhalb des repräsentativen Systems ausgelotet: wie lässt sich die Repräsentationsbasis einerseits und die Stellung der Repräsentanten andererseits verbessern, um Legitimation zu stärken (C.)? In einem weiteren Schritt soll das existierende demokratische System auf anderer Ebene weitergedacht werden und es wird gefragt, inwieweit mehr Partizipation helfen kann, die Legitimation des Staates zu stärken (D.), bevor schließlich ein zusammenfassender Ausblick gewagt wird (E.). 


\section{B. Diagnose: Der Legitimationsverlust des Staates}

Der Legitimationsverlust des Staates lässt sich auf viele Faktoren zurückführen, die teils unabhängig voneinander existieren, in weitaus größerem Maße aber miteinander verbunden sind und sich gegenseitig verstärken. Dazu gehören Internationalisierung und Europäisierung, also die zunehmende Macht internationaler Institutionen wie der WTO oder der EU, die selbst nicht vollumfänglich den tradierten Anforderungen an eine demokratische Ordnung genügen; Privatisierungen und der damit einhergehende Rückzug des Staates und der zunehmende Einfluss nicht-staatlicher Akteure wie der (Finanz-)Wirtschaft; sinkendes Wachstum und steigende sozio-ökonomische Ungleichheit; Kriege und Migration in seit 1945 unbekanntem Ausmaße; der Aufstieg des Parteienstaates; die (behauptete) Dominierung des politischen Prozesses durch nicht gewählte Eliten; die Entfremdung der gewählten Eliten vom Volk sowie der Ausschluss vieler gesellschaftlicher Gruppen aus der Willensbildung in Staat und Gesellschaft. Die Finanz- und Staatsschuldenkrise wirkt wie ein Katalysator für all diese Probleme, die jedoch bereits zuvor erkennbar waren. Angesichts dieser Schwierigkeiten ist dem Staat die Handlungshoheit vielfach entglitten, der Staat stößt an seine Grenzen.

I. Von zentraler Bedeutung ist das Parlament als Herzkammer des demokratischen Staates. Geht das Vertrauen in das einzige unmittelbar gewählte Staatsorgan verloren, so strahlt dies auf das gesamte politische System aus. Die Antworten des Parlaments auf diesen Kontrollverlust stellen aber unter Umständen einen Teil des Problems dar, diagnostiziert Christian Calliess in seinem Beitrag Repräsentanten unter Druck: Zwischen Vertrauensverlust und Ohnmacht. Gesetze zeichnen sich durch eine verstärkte Hinwendung zum Verwaltungsstaat aus, indem Aufgaben auf die Verwaltung und auf Experten übertragen werden. Die Regelungstechnik, derer sich der Deutsche Bundestag bedient, ist die der verstärkten Nutzung unbestimmter Rechtsbegriffe. Die „,nachnormschöpferische Phase“ - d.h. der Vollzug von Gesetzen (im Sinne einer erstmaligen Konkretisierung) im modernen Verwaltungsstaat - stellt aber eine Gefahr dar, da sie die Verwaltung stärkt, die selbst weniger stark legitimiert ist. Außerdem werden häufiger Private in die Normerzeugung involviert. Jedoch handelt es sich dabei keineswegs um die Eröffnung einer Beteiligungsmöglichkeit für alle Bürger, sondern nur für besonders ,interessierte Kreise“ - die selbst nicht demokratisch legitimiert sind. Eine solche Form von „Partizipation“ ist offensichtlich weniger Lösung als eher Teil des Problems. Christian Calliess 
belässt es nicht bei einer Diagnose, sondern bietet erste Lösungsvorschläge für eine Stärkung des Vertrauens der Bürger in das Parlament an. Wesentlich ist, dass das Parlament den von der Verfassung vorausgesetzten und verlangten Einfluss behält und ausfüllt, u.a. indem der Gesetzgeber mit Hilfe seiner Gesetze sicherstellt, dass die Verwaltung etwa private Normgebung - z.B. von Ratingagenturen - nicht kritiklos übernimmt. Auch muss das Parlament eigenständig über wesentliche Fragen, etwa die Euro-Rettung, entscheiden. Vor allem aber folgt aus der Überlassung von Entscheidungsbefugnissen an die Verwaltung, dass als Ausgleich für die Absenkung des materiellen Schutzes des Einzelnen - werden Gesetze immer abstrakter, so gewähren sie auch weniger subjektive materielle Rechte - die Verfahrensrechte des Einzelnen und damit die Partizipation gestärkt werden muss.

Auch in Südafrika schwindet die Kraft des Parlaments als Repräsentationsorgan. Die südafrikanische Demokratie steht im Vergleich zu Deutschland darüber hinaus vor der besonderen Schwierigkeit, dass die Verfassung erst 20 Jahre jung und faktisch davon beeinflusst ist, dass viele Bürger sozio-ökonomisch ausgegrenzt sind. Das Versprechen von materieller Gleichheit durch Demokratie konnte für weite Teile der Bevölkerung noch nicht eingelöst werden. Die andauernde wirtschaftliche Ausgrenzung, zusammen mit einem reduktionistischen Verständnis von Demokratie als bloßem Wahl-Prozess, führt zu Unzufriedenheit gegenüber dem repräsentativen System. Das Parlament wird in Südafrika als verlängerter Arm der herrschenden Partei ANC betrachtet. Korruption grassiert, bestechliche Abgeordnete scheinen mehr Erfolg auf den Wiedereinzug in das Parlament zu haben als nicht korrupte Abgeordnete. Christi van der Westhuizen wirft in ihrem Kommentar Democratising South Africa: Towards a 'Conflictual Consensus' die Frage auf, inwieweit die Idee der Repräsentation wie sie üblicherweise in den westlichen Demokratien verstanden wird Quelle der Unzufriedenheit sein kann. Kritikpunkt ist der punktuelle, nur in mehrjährigem Abstand wiederkehrende Bürgereinfluss und die insgesamt schwache Rückkopplung zwischen Repräsentierten und Repräsentanten. Das Verfassungsprinzip der Responsivität (s. hierzu auch den Beitrag von Barbara Loots) könne zwar Abhilfe schaffen, aber nicht, wenn es bloß formalistisch und ohne Überzeugung angewendet werde. Anschaulich verdeutlicht Christi van der Westhuizen anhand eines aktuellen Beispiels, das Korruption, den Wahlerfolg einer populistischen Partei und den unkonventionellen Umgang mit parlamentarischen Regeln verbindet, die Schwierigkeiten der jungen parlamentarischen Demokratie in Südafrika. Aufgrund 
ihres Wahlerfolgs sind die Economic Freedom Fighters (EFF), eine linkspopulistische Partei, seit 2014 erstmals im südafrikanischen Parlament vertreten. Die Partei bricht in vielerlei Hinsicht mit den jungen parlamentarischen Traditionen. Entsprechend der Schlussfolgerung des „Public Protector" in dem Bericht zum privaten Hausausbau des Staatspräsidenten Jacob Zuma mit Steuergeldern forderten Abgeordnete der EFF immer wieder die Rückzahlung der Kosten i.H.v. umgerechnet 18,7 Millionen Euro. Für diese im Parlament vorgebrachte Forderung wurden EFF Parlamentarier schon mehrfach von der Polizei unter Anwendung von Zwang aus dem Parlament entfernt. Selbst wenn die Abgeordneten zugegebenermaßen die Geschäftsordnung des Parlaments weit auslegten, führt dieser Umgang dazu, dass Wählerinnen und Wähler sich von Politik und repräsentativer Demokratie abwenden. Hierbei handelt es sich jedoch weniger um ein politisches Desinteresse als vielmehr eine Institutionenverdrossenheit. Entsprechendes dürfte sich auch für Deutschland nachweisen lassen, wo seit längerem anerkannt ist, dass der bekannte Begriff der „Politikverdrossenheit" nicht ganz treffend gewählt ist. Trotz der Erfolge des ANC in der Überwindung der Apartheid und eines vergleichsweise friedlichen und fairen Übergangs zu einem demokratischen Gemeinwesen, werden der ANC und sein oft eigennütziger Umgang mit Recht und Gesetz für die Verdrossenheit der Bürger mit der Demokratie verantwortlich gemacht.

Jan Philipp Schaefer sieht in seinem Beitrag Perspektiven der repräsentativen Demokratie den repräsentativen demokratischen Staat westlicher Prägung nach wie vor als weltweites Leitbild guter staatlicher Ordnung an. Dies gelte obgleich einzelne Einwände sog. postdemokratischer Kritiker berechtigt seien, die richtigerweise auf Schwächen in der Repräsentation und damit der Legitimation verwiesen.

II. Obgleich schon aufgezeigt wurde, dass die Gründe für den Legitimationsverlust vielfältig sind, sticht einer hervor, da er ein Querschnittsthema betrifft: mangelnde Transparenz. Es wird oft vorgebracht, dass ohne Transparenz kein Vertrauen entstehe oder es verloren ginge. Nicht nur lässt sich mit Transparenz Korruption bekämpfen. Mit Transparenz können Bürgerinnen und Bürger zumindest in die Lage versetzt werden, sich ein Bild von den politischen Problemen zu machen und auf diese Weise sowohl ihre eigene Erwartungshaltung als auch die Leistungen der Politik kritisch zu überprüfen. Zudem ist die Herstellung von Transparenz eine Frage der rechtlichen Ausgestaltung und somit durch den Staat selbst leistbar. Der Verweis auf externe Faktoren, wie „die Märkte“, „die Globalisie- 
rung“ oder andere nicht beeinflussbare Aspekte, verfängt an dieser Stelle nicht.

Obwohl das Arkanprinzip ein wesentlicher Faktor ist, der zum Schwund von Legitimation beiträgt, darf Transparenz als Voraussetzung einer funktionierenden Demokratie nicht einseitig und überhöht betrachtet werden, betont Jonathan Klaarens Beitrag The South African 'Secrecy Act': Democracy Put to the Test. Er nennt als wesentliche Gründe für Defizite in der repräsentativen Demokratie Südafrikas die fehlende Verantwortung von politischen Eliten, eine unzureichend ausgefüllte Rolle des National Council of Provinces als zweiter Parlamentskammer (vergleichbar dem deutschen Bundesrat), dem es nicht gelingt, die Debatten der lokalen und regionalen Ebene auf ein nationales Niveau zu heben, und schließlich eine unzureichende Kontrolle der Geheimdienste. Klaarens Argument für einen abwägenden Umgang mit Transparenz stützt sich auf die folgenden Überlegungen: Erstens kann auch in einem an sich transparenten System Vertrauen zerstört werden, etwa durch Fehlinformationen und Täuschungen. Zweitens kann Transparenz ebenfalls negative Auswirkungen zeitigen, etwa dadurch, dass sich politisches Handeln informalisiert und gänzlich der Kontrolle entzieht. Eine andere Gefahr liegt darin, dass zu viel Hoffnung in Transparenz gesetzt wird. Ein Beispiel aus den Vereinigten Staaten zeigt dies treffend: Die Entscheidung „Citizens United“ des U.S. Supreme Court aus dem Jahr 2010 hat die Schleusen für die Parteienfinanzierung so weit geöffnet wie nie zuvor. Hier hat der Supreme Court unbeschränkte Ausgaben von juristischen Personen für politische Zwecke, d.h. vor allem Wahlkampfunterstützung, erlaubt. Als Schranke für ungezügelten Einfluss von Partikularinteressen auf die Politik benannte das Gericht die zügelnde Kraft von Transparenz. Die Praxis hat indes gezeigt, dass diese Schranke illusorisch ist und die Folge des Urteils ein weitaus höherer Einfluss von Partikularinteressen als je zuvor ist.

In Südafrika wird seit Jahren keine Debatte um einen Gesetzentwurf intensiver und heftiger geführt als die um die 2014 vom Parlament verabschiedete „Protection of State Information Bill“, von den Gegnern auch „Secrecy Bill“ genannt. Dieser Gesetzentwurf schränkt zwar einerseits Transparenz und Öffentlichkeit staatlichen Handelns ein und stellt damit nach Ansicht der Kritiker die Voraussetzungen einer funktionierenden Demokratie in Frage. Andererseits begrenzt er die Anzahl staatlicher Dokumente, die geheimhaltungsbedürftig sind im Vergleich zur bisherigen Rechtslage. So soll Demokratie gestärkt werden und der verfassungsrechtliche Auftrag, das Recht auf Zugang zu staatlichen Dokumenten positiv- 
rechtlich auszugestalten, erfüllt werden. Der Gesetzentwurf wurde im September 2013 von Staatspräsident Jacob Zuma an das Parlament zurückverwiesen. Es wird allgemein erwartet, dass im Falle der Verkündung des Gesetzes letztlich das Verfassungsgericht entscheiden wird. Wie in einem Brennglas bündeln sich bei diesem Gesetzgebungsverfahren die mangelhafte Partizipation der Bürger, die Rolle der Medien und ihr Kampf um Macht und Einfluss sowie die mangelnde individuelle Verantwortlichkeit der gewählten Repräsentanten in einem System mit einer herrschenden Partei, die jedoch in zunehmendem Maße um ihre Dominanz fürchten muss.

Ein ausdrücklicher verfassungsrechtlicher Transparenz-Auftrag besteht in Deutschland nicht. Gleichwohl beruht die parlamentarische Demokratie auch in Deutschland auf einer öffentlichen Diskussion, der Freiheit der Meinungen und dem Zugang $\mathrm{zu}$ Informationen. Transparenz ist somit nicht nur ein rechtsstaatliches Instrument, sondern auch eine unabdingbare Voraussetzung für die Demokratie. Dabei ist aber ein Ausgleich von Transparenz und Geheimhaltungsinteressen zu schaffen. Elke Gurlit argumentiert in ihrem Kommentar Das Spannungsfeld von Transparenz und Geheimhaltung im demokratischen Staat, dass das Informationsfreiheitsgesetz (IFG) von 2005 diesen Ausgleich nicht vollständig erreicht und staatlichen Geheimhaltungsinteressen ein zu großes Gewicht zumisst, wie u.a. die Bereichsausnahmen für die Geheimdienste belegen. Gefahren für die Demokratie bestehen aber nicht nur durch den Staat selbst, sondern auch durch private Akteure. Insbesondere Unternehmen wie Facebook, Google und Co. stellen aufgrund ihrer marktbeherrschenden Stellung sowie ihres Wissens über jeden Einzelnen eine Bedrohung für individuelle Freiheiten dar. Hierauf muss der Staat reagieren. Auch dies kann Legitimation von Staatsgewalt erhöhen. Konsequenterweise plädiert Elke Gurlit daher für eine umfassende Informationsrechtsordnung. In beiden Ländern zeigt sich, dass noch Spielraum für Verbesserungen in diesem Bereich besteht.

Die Diagnose des Legitimationsverlustes vermengt sich im ersten Teil des Bandes schon mit den ersten Heilungsvorschlägen. Sie zielen dabei sowohl auf Verbesserungen des repräsentativen Systems als auch seiner Weiterentwicklung durch vermehrte Möglichkeiten der Partizipation ab. Verbesserungen und Weiterentwicklungen stärken die Legitimation staatlicher Gewalt. Es kommt vor allem auf das „Wie“ sowie das richtige Maß an: ein Zuviel an Transparenz kann Legitimation ebenso schwächen wie es ein Zuwenig kann. Das Gleiche gilt für ein Zuviel - oder ein Zuwenig - an 
Entscheidungsspielräumen für die Verwaltung oder ein Zuviel an Einfluss von Partikularinteressen. Das richtige Maß ist jeweils Voraussetzung für ein gelungenes demokratisches Gemeinwesen.

\section{Reformmöglichkeiten: Wege zu verbesserter Repräsentation}

Der Stärkung des repräsentativen Systems selbst scheint in der wissenschaftlichen Diskussion weniger Beachtung geschenkt zu werden. Vielmehr überwiegen die Rufe nach mehr direkter Demokratie und Partizipation. Diese einseitige Fokussierung stellt allerdings eine Vernachlässigung des Fundaments jeder westlichen Demokratie dar. Nur wenn die Basis und das ist die Repräsentation - trägt, kann der Staat, wie wir ihn heute kennen, bestehen bleiben. Anderenfalls droht tatsächlich das Ende des repräsentativen Staates. Zu den Ansätzen, die zur Stärkung staatlicher Legitimation beitragen können, gehört - mit Blick auf die Repräsentierten die Ausweitung des Wahlrechts und - mit Blick auf die Repräsentanten die Stärkung der Rechte des Abgeordneten gegenüber seiner Fraktion und dem Parlament. Schließlich ist zu erwägen, ob nicht auch im repräsentativen System der Gesetzgebungsprozess selbst mit partizipativen Elementen angereichert werden sollte, um so das repräsentative System zu stärken.

I. Eine Reformmöglichkeit stellt die Ausweitung des Wahlrechts dar, indem es von der Staatsangehörigkeit abgekoppelt wird und damit Rechtsunterworfene allein aufgrund ihrer territorialen Betroffenheit wahlberechtigt werden. Entsprechende Reformgesetze gab es 1989 in Schleswig-Holstein und in Hamburg sowie zuletzt im Jahr 2013 in Bremen. Die entsprechenden Gesetze wurden vom Bundesverfassungsgericht bzw. dem Staatsgerichtshof für die Freie Hansestadt Bremen als verfassungswidrig verworfen. Von vielen wird angesichts einer global erhöhten Mobilität gefordert, dass das als nationalistisch und anachronistisch empfundene Kriterium der Staatsangehörigkeit nicht mehr die entscheidende Grundlage des Wahlrechts sein dürfe. Spiegelbildlich wird in Betracht gezogen, ob nicht auch eine Einschränkung des Wahlrechts die Legitimation des Staates erhöhen könne, indem im Ausland lebende Staatsangehörige - entgegen der in Deutschland sowie in Südafrika jeweils verfassungsgerichtlich sanktionierten Rechtslage - von der Wahl ausgeschlossen werden. Dies wäre insbesondere dann konsequent, wenn allein die territoriale Betroffenheit für das Innehaben des Wahlrechts entscheidend wäre. Die territoriale Rechtsunterworfenheit wäre dann sowohl positiv als auch negativ konstituierend. 
Diese Positionen, die Wessel le Roux in seinem Beitrag Migration, Representative Democracy and Residence Based Voting Rights in Post-Apartheid South Africa and Post-Unification Germany (1990-2015) einnimmt, sind in Deutschland und Südafrika äußerst umstritten, wenngleich in der ersten Wahl Südafrikas nach dem Ende der Apartheid 500.000 Ausländer wählen durften. Die Europäische Kommission für Menschenrechte stellte schon 1997 eine Korrelation zwischen der Betroffenheit des Einzelnen und seinem Wahlrecht her; der Europäische Gerichtshof für Menschenrechte hat diese Rechtsprechung später mehrfach bestätigt. Es ging hierbei jedoch immer um die Einschränkung des Wahlrechts von Staatsangehörigen, die im Ausland ansässig waren. Dieser Ansicht lässt sich entgegenhalten, dass weiterhin ein Unterschied zwischen der Bevölkerung und dem Volk gemacht werden müsse, da nur eine Einheit repräsentiert werden könne und keine Vielheit. Loyalitätskonflikte seien vorprogrammiert und solche könne sich eine Gesellschaft, die am Ende ihres „Leben[s] im politischen Schlaraffenland" angekommen sei, nicht leisten, so der vehemente Widerspruch von Otto Depenheuer in seinem Kommentar Ende der repräsentativen Demokratie? Eine Staatsform vor der Alternative ihrerselbst.

II. Richtet man den Blick auf den einzelnen Abgeordneten, so stellen sich die Fragen nach seiner Stellung zum Parlament und zur Fraktion. In einer Parteiendemokratie, wie Südafrika und Deutschland es sind, manifestieren sich die Probleme des einzelnen Abgeordneten besonders im Hinblick auf seine Stellung zur eigenen Fraktion. Hier gilt: je stärker die Fraktion, desto schwächer der Abgeordnete. Richard Calland und Shameela Seedat betonen in ihrem Beitrag Institutional Renaissance or Populist Fandango? The Impact of the Economic Freedom Fighters on South Africa's Parliament, wie wichtig das parlamentarische Leben für die Legitimation staatlichen Handelns ist und beleuchten so die alte Konfrontation zwischen Redeparlament und Arbeitsparlament neu. Die Autoren nutzen hierfür wie Christi van der Westhuizen das Beispiel der EFF, deuten es aber weitaus positiver. Ihrer Auffassung nach haucht deren Umgang mit den Parlamentsregeln neues Leben in die etablierte Struktur des südafrikanischen Parlaments ein. Ein Bedeutungszuwachs des Parlaments gegenüber der dominierenden Partei des ANC werde sicher stattfinden, auch wenn es sich bei der EFF ironischerweise um eine prinzipienlose und populistische Partei handelt und der Bedeutungszuwachs noch Zeit brauche. Trotz Schwächen der südafrikanischen Verfassung dürfte die Lösung der Probleme weniger in einer Änderung des geschriebenen Verfassungsrechts liegen als vielmehr in einer Änderung der ökonomischen und sozialen Le- 
bensbedingungen und der Fähigkeit der Oppositionsparteien, die Exekutive zu kontrollieren und verantwortlich zu halten.

Auch in Deutschland fordert das Verhalten der etablierten und im Bundestag organisierten Parteien Kritik heraus, so etwa wenn aus machtpolitischen Erwägungen ein nicht verfassungsgemäßes Wahlrecht beschlossen wird. Der Staat wird hier zur Beute, so heißt es immer wieder. Für die Wiederbelebung des repräsentativen Systems ist ebenso wichtig, über das „Wie“ - und nicht lediglich das „Ob“ - einer Neuregulierung der Parteienfinanzierung und die Stärkung oppositioneller Minderheitenrechte gerade in Zeiten einer übermächtigen „Großen Koalition“ nachzudenken. Auf diese und andere Probleme geht aus deutscher Sicht Sophie-Charlotte Lenski in ihrem Kommentar Abgeordnete zwischen Parteibindung, Regierungsdisziplin und neuen Formen der Partizipation ein. Sie sagt, anders als es Richard Calland und Shameela Seedat für Südafrika tun, für Deutschland in der nächsten Zeit eine Schwächung und keine Stärkung des Parlaments voraus. Insbesondere der einzelne Abgeordnete - gleichgültig, ob als Teil einer Regierungs- oder einer Oppositionsfraktion - verliert an Einfluss und Bedeutung. Aber auch wenn man den parlamentarischen Raum im engeren Sinne verlässt und das Verhältnis von Partei, Staat und Gesellschaft betrachtet, ist derzeit nicht alles zum Besten bestellt. Nunmehr bemächtigen sich auch die Fraktionen der gesellschaftlichen Sphäre durch ein stetes Mehr an Öffentlichkeitsarbeit, wobei sich z.B. die Öffentlichkeitsarbeit der - öffentlich alimentierten - Regierungsfraktionen nicht wesentlich von der Öffentlichkeitsarbeit der Regierung selbst unterscheidet. Fraktionsmitglieder und deren Mitarbeiter werden vorausschauend mit öffentlichen Ämtern oder Beamtenstellen versorgt, bevor der Ausgang der nächsten Wahl diese Personen ,unversorgt“ lässt. Diese Mechanismen lassen den Legitimationsstrang, der vom Volk zum gewählten Abgeordneten führen sollte, zerfasern. Ist jedoch der Wählerwille immer weniger von Bedeutung für den Abgeordneten aufgrund anderer Abhängigkeiten, so kann dies nicht ohne Auswirkung auf die Repräsentation bleiben.

III. Eine weitergehende Stärkung des repräsentativen Systems - bereits an der Grenze zur Einführung direktdemokratischer Elemente auf Bundesebene (D.) - kann auch die Einführung neuartiger partizipativer Elemente in das repräsentative Gesetzgebungsverfahren sein. Bislang finden in den Bundestagsausschüssen gem. $\S 70$ der Geschäftsordnung des Bundestages nur Anhörungen von Experten und Interessensvertretern statt. Das Volk wird indes nicht unmittelbar angehört. Dies ist in Südafrika anders. Zwei 
wegweisende Urteile des südafrikanischen Verfassungsgerichts zum verfassungsrechtlich normierten, aber bis dahin kaum konturierten Prinzip der Responsivität staatlichen Handelns haben dazu geführt, dass das Volk in stärkerem Maße als in Deutschland in den parlamentarischen Gesetzgebungsprozess einbezogen ist. Das Prinzip der Responsivität staatlichen Handelns sieht eine Pflicht des Staates vor, die Bürger einzuladen, sich zu Gesetzesvorhaben zu äußern und diese Äußerungen aufzunehmen und zu berücksichtigen. Verstöße gegen dieses Prinzip haben schon zur verfassungsgerichtlich festgestellten Nichtigkeit von Gesetzen geführt. Barbara Loots stellt in ihrem Beitrag Civic Dignity as the Basis for Public Participation in the Legislative Process dieses verfassungsrechtliche Instrument, insbesondere seine Möglichkeiten und Grenzen dar. Sie betont, dass das Prinzip der Responsivität eines der zentralen Elemente der südafrikanischen Verfassung darstelle, auf die Würde des Bürgers zurückgeführt werden könne und das Ziel verfolge, angesichts des Apartheid-Regimes und der damaligen nahezu unbegrenzten Stellung und Macht des Parlaments dieses unter demokratische Kontrolle zu bringen. Mit dem Prinzip der Responsivität stellt die Verfassung sicher, dass Demokratie kein leeres Versprechen bleibe und Menschenrechte - anders als vor 1994 - geschützt werden.

Dass diese Ideen auch auf Deutschland übertragen werden können, zeigt die Landesebene. So hat das Prinzip der Responsivität in BadenWürttemberg Anklang gefunden - wenngleich ein direkter Normentransfer nachweislich nicht stattgefunden hat: in Südafrika ist die Beteiligung zwingend, in Baden-Württemberg lediglich ein freiwilliges Experiment, aber dennoch ein höchst beachtenswertes. Das Land ist in Deutschland Vorreiter in Fragen der Bürgerbeteiligung bei Gesetzgebungsverfahren, die Methoden hinsichtlich der Gesetzgebung ähneln dem südafrikanischen Beispiel. Hier werden die Bürger dazu eingeladen, ihre Meinungen online in den Gesetzgebungsprozess einzubringen. Diese Beiträge werden gelesen und bewertet. Sie finden im Gesetz ebenso Berücksichtigung wie Expertenmeinungen. Fabian Reidinger fragt in seinem Kommentar BadenWürttemberg zwischen Wählen, Mitreden und Entscheiden - Mehr Partizipation als Regierungsauftrag, ob dadurch die Akzeptanz von Gesetzgebungsvorhaben in der Bevölkerung steigt oder ob womöglich - im Gegenteil - eine Politisierung zu einer Polarisierung in der Bevölkerung führt und beleuchtet, wie die Abgeordneten - und die Ministerien - dieses „neue“ Instrument annehmen und wie es insoweit zu einer Veränderung der politischen Kultur kommt. Dass dieses Instrument der Bürgerkonsulta- 
tion gar nicht revolutionär ist, belegt das Grußwort von Cosima Möller. Sie verweist auf die Entstehungsgeschichte des Allgemeinen Landrechts für die preußischen Staaten von 1794. Während der langjährigen Vorbereitungen wurden nicht nur Experten an der Ausarbeitung des Gesetzes beteiligt, sondern wurde auch die Bevölkerung zu Vorschlägen ermuntert.

\section{Weiterentwicklung des Systems: Elemente direkter Demokratie und ihre Auswirkungen}

Abschließend geht es um die Stärkung der Demokratie durch die Anreicherung des repräsentativen Systems mit direktdemokratischen und partizipatorischen Elementen. Der Begriff der direkten Demokratie bezieht sich traditionell auf die Gesetzgebungsebene. Auf Ebene der Verwaltung wird hingegen zumeist von partizipativer oder deliberativer Demokratie gesprochen. Alle Formen zeichnen aus, dass die Staatsgewalt in einem offenen Prozess an die Gesellschaft rückgebunden wird. Durch Deliberation wird neben der Rückbindung der Staatsgewalt an die Bürger auch ein Interessenausgleich ermöglicht: der Verantwortungszusammenhang zwischen Staatsgewalt und Gesellschaft wird gestärkt, indem der Staat den Bürgern antworten muss. Beteiligung dient außerdem auch der (demokratischen) Kontrolle des Staates durch die Bürger. So verstanden, können die Elemente direkter Demokratie neben der Legislative auch die Beteiligung auf Exekutivebene umfassen.

I. Das Primärbeispiel von Bürgerbeteiligung ist die direkte Demokratie als Form der Gesetzgebung durch Referenda und Volksinitiativen. Obgleich die Diskussion schon länger geführt wird, sah man in Deutschland etwa durch die Koalitionsverhandlungen 2013 und die unterschiedlichen Positionen für die Bundesebene von CSU und SPD einerseits und CDU andererseits die Aktualität dieser Fragen. Außerdem hat Deutschland durch die Einführung und vermehrte Nutzung von Volksgesetzgebung in allen 16 Ländern neue Erfahrungen gesammelt. Sie birgt Chancen und Risiken - die Chancen überwiegen jedoch die Risiken: Voraussetzung dafür ist zunächst, dass repräsentative Demokratie und Parlamentarismus weiter als der Regelfall verstanden werden, betont Peter M. Huber in seinem Beitrag Direkte Demokratie? Gefahren und Chancen für das repräsentative System. Direkte Demokratie ergänzt das bestehende System, ersetzt es aber nicht. Schon die geringe Anzahl an erfolgreichen Volksbegehren auf Landesebene zeigt, dass die beschworenen Gefahren wohl geringer als be- 
fürchtet sind. Der Nutzen hingegen ist groß. So wirkt direkte Demokratie verzögernd gegenüber dem Druck durch die Internationalisierung und Privatisierung. Außerdem stellt sie ein Gegengewicht zum Parteienstaat dar, der ebenfalls zu den Auslösern eines tiefen Skeptizismus gegenüber dem repräsentativen System zählt. Elemente direkter Demokratie erhöhen Akzeptanz und bringen Rechtsfrieden und werden daher auch auf Bundesebene dringend benötigt.

Eine ähnliche Diskussion zu direkter Demokratie wird in Südafrika nicht geführt. Das mag daran liegen, dass die Verfassung einerseits ein höheres $\mathrm{Ma} ß$ an Beteiligung und Responsivität garantiert, dass aber andererseits dieser verfassungsrechtliche Anspruch in der Realität ohnehin nicht eingelöst wird, wie die zahlreichen Proteste belegen. Das Alltagsleben vieler Südafrikaner ist nach wie vor beeinträchtigt durch Armut, Ungleichheit, soziale Exklusion, mangelnde öffentliche Daseinsvorsorge und Korruption.

II. Sowohl die mögliche Einführung direkt-demokratischer Elemente auf der Ebene der Bundesgesetzgebung als auch der Verwaltungsebene bestimmen die Partizipationsdiskussion der letzten Jahre. Gerade Planungsverfahren für infrastrukturelle Großprojekte (etwa Stuttgart 21, Transrapid, Stromleitungs-Trassen, Kohlekraftwerke) rufen den Widerstand der Bürger hervor. Wesentliche Beachtung hat deshalb Beteiligung im Planungsrecht gefunden, welches den Bürger oft unmittelbarer betrifft als das Handeln der Regierung oder des Parlaments. Dies mag erklären, weshalb die Bürgerbeteiligung an Rechtsverordnungen, die den Großteil der Gesetze ausmachen, dogmatisch unterbeleuchtet ist. Zwar unterscheidet sich Partizipation rechtlich wesentlich danach, ob materiell-rechtliche Gesetze oder Einzelakte erlassen werden sollen. Die tatsächlichen Erwartungen an Partizipation sind aber sehr ähnlich. So geht es um Effektivität der Rechtsverwirklichung, Information, Transparenz, Kontrolle, Akzeptanz und die Kompensation von Defiziten der repräsentativen Demokratie. Diese Erwartungen sind jedenfalls teilweise zu hoch, warnt Jan Ziekow in seinem Aufsatz Exekutive Entscheidungen und Partizipation: Verbesserung der Steuerungsfähigkeit des Staates und der Legitimität staatlichen Handelns? Er führt aus, dass allenfalls eine Verfahrens-, aber keine Ergebnisakzeptanz erwartet werden könne. Und auch diese stellt sich nur bei „gut“ durchgeführter Partizipation ein. Damit ist das Kernproblem angesprochen, dass nur die „richtige“ Partizipation die an sie gestellten Erwartungen erfüllen kann, aber „schlecht“ vorbereitete und/oder durchgeführte Partizipation die gegenteiligen Effekte bewirken kann. Kriterien sind Ziel- 
klarheit und Formenadäquanz, Konfliktanalyse, Zeitgerechtigkeit, die Grundsätze der Informiertheit, Fairness und Transparenz sowie Responsivität. Während Partizipation bei Einzelentscheidungen im Verwaltungsverfahren gesetzlich vielfach vorgesehen ist, fehlt es bei Rechtsverordnungen in Deutschland an einer systematischen Einbeziehung der Öffentlichkeit. Eine Stärkung dieser Form der Beteiligung ist verfassungsrechtlich begrenzt: es kann sich immer nur um eine Mitberatung handeln, nicht um Mitentscheidung. Ansonsten würde die im Grundgesetz vorgesehene Verantwortung der Regierung konterkariert werden. Bei der Beurteilung von Partizipation auf Rechtsverordnungsebene ist stets zu beachten, dass eine gesetzliche Grundlage besteht und verfassungsrechtlich bestehen muss. In Abhängigkeit davon, wie stark die gesetzliche Determination ist, strahlt die legitimierende Kraft des Gesetzes in stärkerem oder in schwächerem Maße auf die Rechtsverordnung ab. Gerade in Regelungszusammenhängen die eine hohe gesellschaftliche, ökonomische und/oder wissenschaftlich-technologische Dynamik mit einer entsprechenden Komplexität des Regulierungsrahmens und Ungewissheit der Entscheidungsgrundlagen aufweisen, liegt regelmäßig nur eine geringe gesetzliche Determinierung vor. Insbesondere hier bleibt Raum für die legitimierende Kraft der Partizipation, die ergänzend, aber nicht ersetzend wirkt.

Gespiegelt wird der Aufsatz von Petrus Maree, Participation in Executive Rule-Making: Preliminary Observations towards a Conceptual Framework, der u.a. die Frage aufwirft, weshalb Partizipation eine Wirksamkeitsvoraussetzung von Gesetzen, aber nicht von Rechtsverordnungen ist. Er sieht in Südafrika Verbesserungsbedarf hinsichtlich der Bürgerbeteiligung bei Verwaltungsentscheidungen im Unterschied zur Beteiligung der Bürger am Gesetzgebungsverfahren. Er konstatiert für Südafrika - insoweit übereinstimmend mit den deutschen Erfahrungen - bereits einen unzureichenden Zugang zu Verwaltungsinformationen und fordert einen erleichterten Zugang und eine verbesserte Verwaltungskultur.

III. Bislang fehlt es an einem umfassenden und systematischen Konzept zur rechtlichen Erfassung des Wandels der Staatsgewalt hin zu mehr Partizipation. Abschließend stellen sich daher zwei Fragen: Wer ist an der Ausübung öffentlicher Gewalt zu beteiligen? Und: Welche Rechtswirkung soll von der Beteiligung ausgehen? Hierfür müssen alle drei Staatgewalten in den Blick genommen werden. Dabei zeigt sich, dass die verschiedenen Funktionen von Partizipation mit denen der Gewaltenteilungsebene überlappen. Sowohl die Partizipation als auch die Gewaltenteilung beruhen auf dem Demokratieprinzip, dem Rechtsstaatsprinzip und dem Effektivitäts- 
prinzip. Der Gewaltenteilungsgrundsatz löst darüber hinaus das zwischen Demokratie- und Rechtsstaatsprinzip bestehende Spannungsverhältnis auf, indem er die verschiedenen Staatsaufgaben bestimmten Organen zuordnet. Dominik Steiger argumentiert in seinem Aufsatz Gewaltenteilung als Mittel zur Konzeptualisierung von Partizipation, dass der Gewaltenteilungsgrundsatz auch im Hinblick auf Partizipation die Spannung beider Prinzipien auflösen kann und beide Fragen beantwortet: Auf der Ebene der Judikative dürfen sich grundsätzlich nur Einzelne beteiligen, die in ihren Rechten betroffen sind. Die Entscheidungsmacht verbleibt beim Gericht. Auf Legislativebene muss das Volk neben Personal- auch Sachfragen entscheiden können. Auf Ebene der Exekutive bedarf es für die Bestimmung des Ausmaßes des Einflusses von Partizipation einer genauen Analyse, ob Rechtsstaatlichkeit oder demokratische Willensbildung im Vordergrund stehen. Die Entscheidung wird unabhängig davon immer vom staatlichen Organ getroffen, aber es dürfen sich mal mehr, mal weniger Bürger beteiligen.

Eine solche Theorie der Beteiligung lässt sich auch auf Südafrika und die Rechtsprechung des südafrikanischen Verfassungsgerichts übertragen. Henk Botha argumentiert zusätzlich - entsprechend dem Titel seines Kommentars Democratic Participation and the Separation of Powers -, dass der demokratische Charakter von Partizipation in Südafrika stärker betont werde als im deutschen Kontext, wo Partizipation oftmals sogar als anti-demokratisch verstanden wird. Henk Botha macht jedoch darauf aufmerksam, dass sozio-ökonomische Realitäten nicht ausgeblendet werden dürfen. Partizipationsmöglichkeiten und ihr Erfolg hängen von der Verfügbarkeit von Ressourcen wie Zeit, Geld oder Vertrautheit mit rechtlichen Prozessen ab. Arme und Benachteiligte laufen somit Gefahr, kein Gehör zu finden, welches dem Gewicht und der Dringlichkeit der Anliegen entspricht. Die Grenze zwischen individueller und kollektiver Beteiligung muss deshalb so gezogen werden, dass sie die Effekte tiefsitzender struktureller Ungleichheit berücksichtigt. So sei der Einfluss der Gerichte auf die Ermöglichung demokratischer Willensbildung und auf kollektive Selbstbestimmung als Korrektiv zum Parlament weitaus wichtiger als in Deutschland. Ebenso sei aufgrund der vielen Minderheiten in Südafrika sicherzustellen, dass diese besondere Partizipationsmöglichkeiten in Gesetzesverfahren Eingang erhielten, die sie besonders beträfen. 


\section{E. Fazit}

Die hier von deutschen und südafrikanischen Referentinnen und Referenten vorgelegten Aufsätze, die jeweils aus ihrem Land und ihrer Perspektive die Probleme und entsprechende Lösungsmöglichkeiten darlegen und diskutieren, zeigen, dass die Debatte in den verschiedenen Bereichen unterschiedlich weit fortgeschritten ist. Beide Staaten könnten somit von den Erfahrungen der jeweils anderen Jurisdiktion profitieren.

Gemeinsamkeit besteht insoweit, als die rechtliche Ausgestaltung des repräsentativen Systems zwar bedeutsam, für sich allein jedoch kein hinreichender Garant für eine funktionierende Demokratie ist. Die Lebenswirklichkeit der Bevölkerung muss ebenfalls berücksichtigt werden. Hierzu gehören auch Erwartungen an das System bzw. diesbezügliche Haltungen sowie das Verständnis der Rolle des Individuums. Die Anreicherung der parlamentarischen Demokratie mit zusätzlichen partizipativen Elementen dürfte zum Zwecke der Stärkung von Legitimität vermehrt gefordert und dementsprechend umgesetzt werden. Wie auch Transparenz ist Partizipation - unabhängig davon, ob sie auf legislativer oder administrativer, nationaler oder lokaler Ebene stattfindet - kein Selbstzweck und kein Allheilmittel. Sie ist ein Instrument, um bestimmte Zwecke zu fördern und Ziele zu erreichen. Dazu gehören Demokratie, Rechtsstaatlichkeit und Effektivität staatlichen Handelns. Je nach Kontext kann Partizipation nützlich und sinnvoll sein. Stets muss ihr Einsatz durchdacht sein, sonst werden die geweckten Erwartungen enttäuscht und das Gegenteil dessen bewirkt, was erreicht werden sollte. Auch jenseits der rechtlichen Ausgestaltung der Verfassung sowie der Rechtsordnung insgesamt liegen zu berücksichtigende Gelingensbedingungen. Demokratie ist abhängig von Voraussetzungen und Wirkmechanismen kultureller, politischer, sozialer und ökonomischer Art, welche durch Demokratie weder erzwungen noch dauerhaft garantiert - aber doch beeinflusst werden können.

Die Aufsätze zeigen: Die repräsentative Demokratie hat Zukunft, sie muss sich jedoch weiterentwickeln. Um es im Sinne Giuseppe Tomasi di Lampedusas zu sagen: Wenn wir wollen, dass die Demokratie so bleibt wie sie ist, dann muss sich vieles ändern. Welche Änderungen denkbar, wünschenswert und (verfassungsrechtlich) umsetzbar sind, ist Gegenstand dieses Bandes. 